\title{
ANALYTICAL AND COMPUTER- GRAPHIC METHOD OF SURFACES' FORMATION PROJECTED BY RAYS OF CONGRUENCE OF CYLINDRICAL SCREW LINES WITH THE CONSTANT STEP
}

\author{
Elena Vasilyevna Simenko" ${ }^{* 1}$, Sergei Anatolyevich Ignatiev ${ }^{* 2}$, Marianna Vladimirovna Voronina ${ }^{* 3}$ \\ * Department of Descriptive Geometry and Graphics, Saint-Petersburg Mining University, \\ 2, 21st Line, Saint Petersburg, 199106, Russian Federation \\ ${ }^{1}$ kii-simenko@mail.ru, ${ }^{2}$ isa@spmi.ru \\ 3 maria.vv@mail.ru - correspondent author
}

\begin{abstract}
Relevance of the research: The relevance of this study is due to the fact that the condition for the use of computer technologies in design requires an analytical interpretation of existing constructive ways of shaping products of complex shape. Development of the general analytical model of nonconventional systems of projection and their projecting surfaces applicable in computer technologies makes a current problem. Since screw motion is one of the most "technological" movements after the translational and rotational motion, the problem of computer-aided design of screw surfaces is relevant. This paper presents an analytical interpretation of the method of surface shaping by screw projection of a line from the standpoint of the general theory of analytical shaping. Parametric equations of congruence of coaxial cylindrical helical lines of constant step are given. The path of transition to parametric equations of congruence surfaces are shown, the linear framework of which consists of screw lines projecting any line of space. Purpose of the study: The purpose of this article is to identify features of the system, the projecting rays of which coincide with a trajectory of the most "technological" movement: screw displacement. Methods of research: The leading method of investigating this problem is the general analytical theory of applied surface molding, developed by Professor Skidan I.A. and making up a unified mathematical apparatus for geometric modeling of surfaces, corresponding to modern requirements of use of computer technologies. Results of the research: The parametric equations of congruence of coaxial cylindrical helical lines of a constant step are presented, the transition to parametric equations of congruence surfaces is shown, the linear framework of which consists of screw lines projecting any line of space. Significance of the study: As a result of the research, an analytical interpretation of the method of shaping by screw projection has been developed which allows to take into account such requirements to the surface as passing through a point or line and, what is most important, to apply computer technologies in designing and manufacturing screw-shaped products on equipment with numerical program control (CNC). Conclusions: Analytical interpretation of the method of surface shaping by screw projection of a certain line from the standpoint of the general theory of analytical formation has been developed.
\end{abstract}

Keyword - screw line, screw surface, screw projection, congruence, parametric equations.

\section{INTRODUCTION}

\subsection{Relevance of the problem}

The central and parallel projection due to the fact that at their application the straight line is mapped onto any plane of projections of a straight line, they have the most widespread use. We will call them traditional. The purpose of traditional systems is the graphic modelling of three-dimensional objects by their projections, at least on two planes making the system of projections.

Recently in application-oriented geometry many non-traditional systems of projection are introduced. Emergence of non-traditional systems is caused by need for graphical representation and research of specific objects, in particular, the surfaces formed by the family of rays of the corresponding system, projecting the presented line. Such systems can be classified by type of the ray and by determinant of the system itself, which would provide the passage of one ray through an arbitrary point of the area of space defined for it. In this case, the concept of a projecting ray extends to a planar or three-dimensional curve. In case of systems with rectilinear 
beams, the direction of the ray passing through an arbitrary point is determined using the determinant of the system. The condition of passage through an arbitrary point in the space of a single ray of a non-traditional system leads to the establishment of a unique correspondence between plane fields.

Projecting surfaces are often examined through the study of correspondence, choosing planes of projections so that the singularities of the correspondence lead to features of the surface. On the other hand, using the represented point correspondence of the projection planes, one can imagine a projection system. If we abstract away from the planes of projections and from the point correspondences between them, which is established by a certain unconventional projection system, and take into account that the projecting surfaces of the system can be created by projecting an arbitrary line, then an analytic description of the projecting surfaces can be obtained by assuming that they are surfaces of congruence of projecting rays. In this case, need for projection planes are excessive. The method for shaping surfaces by screw projection of lines was justified in «Screw design and its application for solving geometric and technical problems», «Profiling of conjugate screw surfaces by the method of curvilinear design» and «Screw design» (Tevlin, 1966; Tevlin, et. al., 1968; Podgorny, 1963). The rationale was of a constructive nature, which prevented the use of computer technologies in design.

\subsection{State of the problem development}

- The method of forming surfaces by screw projection of lines was justified in many works. But justifications had constructive character that prevented use of computer technologies in design.

- Analytical interpretations of these methods are known, disaggregated by presentation forms and not associated with input data of software complexes of computer-aided scientific research systems (ASSR), computer-aided design (CAD) systems and automated systems of technological preparation of production (ASTPP).

\subsection{Theoretical and practical contribution of the materials of the article}

- The parametric equations of congruence of coaxial cylindrical screw lines of a constant step are presented, the transition to parametric equations of congruence surfaces is shown, the linear framework of which consists of screw lines projecting any line of space.

- Studies of non-traditional projecting systems and their projecting surfaces were carried out by synthetic or constructive methods.

- As a result of the research, an analytical interpretation of the screw projection forming method that allows to take into account such requirements to the surface as passing through a point or line is developed and, what is most important, to apply computer technologies in the design and manufacture of screw-shaped products on equipment with numerical control (CNC).

- An analytical interpretation of the method for shaping surfaces by screw projection of a line from the standpoint of the general theory of analytical form-formation is developed.

- In the work the analytical interpretation of the method of forming the surfaces by screw projection of a line from the standpoint of the general theory of analytical formation is disclosed.

- Parametric equations of congruence of coaxial cylindrical screw lines of a constant step are given.

- The path of transition to parametric equations of congruence surfaces are shown, the linear framework of which consists of screw lines projecting any line of space.

\subsection{Goals and objectives of the study}

The purpose of this article was to identify the features of the system, projecting rays that coincide with the trajectory of the most "technological" movement: screw displacement.

\section{LITERATURE REVIEW}

Work «Helical Surfaces» in Encyclopedia of Analytical Surfaces presents an all-embracing collection of analytical surface classes. It provides concise definitions and description for more than 500 surfaces and categorizes them in 38 classes of analytical surfaces (Krivoshapko \& Ivanov, 2015).

Authors Krivoshapko S.N. \& Christian A. Bock Hyeng present a review article, contains 45 references, is devoted to an analysis of the literature on the geometric researches of cyclic surfaces with generating circles of constant and various diameters (Krivoshapko \& Bock Hyeng, 2012).

Authors Krivoshapko S.N and Rynkovskaya M. provide an overview of five types of ruled helical surfaces which can be used for helical conveyers, support anchors and screws. The geometry of helical surfaces has been well studied by authors. Several available methods of strength analysis of helicoidal shells give one a choice in solving one-dimensional or two-dimensional problems. Several works dealing with stress-strain state of oblique, pseudo-developable helicoids, and pseudo-developable helicoid of general type are given in references (Krivoshapko \& Rynkovskaya, 2017).

Given a cloud of measurement points from the surface of a 3D object, scientists Pottmann H., Leopoldseder S., Wallner J., Peternell M. have addressed the problem of recognizing and reconstructing 
special surface types. They survey their work on the problem, which is based on approximation in the space of lines and in the space of planes. Moreover, they discuss new generalizations which also use a recently developed technique for parametric surface fitting with an active contour model (Pottman, et. al., 2015).

Scientist Tatiana Olejníková describes method of modelling of cyclical surfaces created by helix on the torus $\Phi$. The axis of the cyclical surface $\Phi^{\prime}$ is the helix $\mathrm{s}$ as a trajectory of movement of a point composed of two motions of rotation. The circle moves together with Frenet-Serret moving trihedron along the helix s and creates the cyclical surface $\Phi^{\prime}$. The paper describes modelling of cyclical surfaces created by moving circles about tangent, principal normal or binormal of the helix s. Paper describes also modelling of triangular grids on the torus. The grids are created by right-handed and left-handed cyclical helical surfaces and by cyclical surfaces with axis on meridians and circles on the torus. Analytic representation, classification of surfaces and some of their geometric properties are derived. (Olejníková, 2009; Olejníková, 2014).

The cylindrical helical surfaces with constant pitch are frequently used in the construction of machine parts which transmit movements (helical teethed wheels, translation screws) or which circulate fluids under pressure (helical pumps, helical compressors). The shells with cyclic middle surfaces are particularly useful as connecting parts of pipelines, in spiral chambers of turbines in hydroelectric power stations, in public and commercial buildings, for example, as coverings of stadiums, in water attractions, and so on.

As it is known cylindrical compression springs are common in modern mechanisms, machines and devices in various industries (Artemyev et al., 2010; Artemyev et al., 2012; Artemyev et al., 2004; Artemyev et al., 1999; Isaev, 2009). And students of engineering sciences should be able to develop such a spring in a variety of CAD systems, gaining experience and skills of modern design with the use of 3D-graphics (Folomkin \& Voronina, 2017). The generation of the helical surfaces is made using tools bounded by surfaces of revolution.

The developed by scientists Saša Ćuković, Goran Devedžić and Ionuţ Ghionea macro has a large number of advantages: direct application in industry for different profiles of helical surfaces, high level of flexibility and interactivity to users and applications, integration with other systems for product development, application in a variety of operating systems, possibility to use it for unlimited number of times, saving time and budget etc. (Ćuković, et. al., 2009).

Bergman, D.L. \& Refael, G. present a theoretical investigation of the nature of «helical metals» bulk metals with helical surface states. They explore how the surface and bulk states can mix, in both clean and disordered systems. Using the Fano model, they have discovered that in a clean system, the helical surface states are not simply absorbed by hybridization with a nontopological parasitic metallic band. Instead, they are pushed away from overlapping in momentum and energy with the bulk states, leaving behind a finite-lifetime surface resonance in the bulk energy band. Furthermore, the hybridization may lead in some cases to multiplied surfacestate bands, in all cases retaining the helicalcharacteristic. Weak disorder leads to very similar effects - surface states are pushed away from the energy bandwidth of the bulk, leaving behind a finite-lifetime surface resonance in place of the original surface states (Bergman \& Refael, 2010).

A dedicated software application, developed by scientists Silviu Berbinschi, Virgil Teodor and Nicolae Oancea allows for modeling of the helical surface. A graphical method presented by them for profiling the shaping tool used to generate cylindrical helical surface with constant pitch. The method, developed in the CATIA graphical design environment, is based on the helical movement decomposition into an assembly of equivalent movements, rotation and translation (Berbinschi, et. al., 2011). The SV \& Toolbox software package, developed by the same authors mutually with Nicuşor Baroiu, offers accessible engineering usability with the scope of building various cutting tools, complex and competitive, through dedicated modules of the software. In the end, the software generates CNC code files and send them in real time to tool-machines with numerical control, in order to follow the movement sequence necessary to mobile elements in the actual fabrication of the cutting tool (Baroiu, et. al., 2012).

Authors Frumusano Gabriel, Silviu Berbinschi and Nicolae Oancea present comparatively two methodologies that can be used in profiling a cylindrical tool reciprocal enwrapped to a cylindrical helical surface with constant pitch: the Rack-tool method, for generating frontal profiles of the helical surface and the Contact line method, for profiling the cylindrical (planing) tool to generate helical slots. Therefore, the use of a simpler method (plane enwrapping) becomes possible to solve spatial enwrapping problems, and the computational volume substantially reduces, by transforming a 3-D enwrapping problem in a plane one (Frumusanu, et. al., 2013).

In the papers of Virgil Teodor, Ionuţ Popa, Gabriel Dura and Nicolae Oancea a solution for the case when the surface to be generated is known only approximately at discrete points is proposed. Bezier polynomials are used to elaborate a specific methodology for profiling tools bounded by primary surfaces of revolution, which generate in the relative motion between the tool and the blank a helical surface. Camelia Popa, Virgil Teodor, Nicusor Baroiu and Nicolae Oancea have proposed an algorithm for polyhedral expression of the helical surface previously determined by reverse engineering methods and an algorithm for the determination of 
the specific enveloping condition at contact with a discrete surface. It is presented an example for a compressor rotor measured on a $3 \mathrm{D}$ measuring machine, the algorithm for the transformation of the gathered points cloud in a surface with polyhedral expression. Given these conditions there were determined the enveloping condition and the axial section of the side mill (Teodor, et. al., 2009; Popa, et. al., 2014).

Scientists Quan-Quan Han and Ri-Liang Liu note, that the helical groove shape plays a key role in ensuring the adequate flute space of many screw components. In many situations, the helical groove is machined through profiled grooving cutter, which brings a huge cost. This study establishes the mathematical model of helical groove based on cross-section and presents an approach to calculate tool path using the whirling process which machines helical groove through enwrapping movements with standard cutters. Finally, a case study and the error analysis are provided to illustrate the validity of the developed models and algorithms, which offers an alternative method for further computer aided manufacturing (Han \& Liu, 2013).

Scientists Denis Znamenskiy and others have developed a topological description of a $\beta$-sheet in terms of a piece of helical surface. It requires only two easy-to-handle parameters: the twist, i.e. the turn of the helical surface per residue, and the coiling, which is a curvature long the strands or in the direction perpendicular to the strands of the sheet. This method applies fairly well to three-and four-strand sheets, forming a too limited structure to be able to build a barrel. From an analysis of $\beta$-sheets derived from a structural database, we show that this picture can even be reduced to the use of one main value, the twist angle. The dependence of $\beta$-sheet twisting on the number of strands in a sheet, and also on the length and direction of strands, has been demonstrated. The applications of such a description may include the rapid modeling of 3D structures (Znamenskiy, 2000).

In the work of scientists Mahmut Ergut, Talat Korpinar and Essin Turhan normal ruled surface of general helices in the Sol space, one of Thurston's eight 3-dimensional geometries, are studied. Also, explicit parametric equations of normal ruled surface of general helices in the Sol space are found (Ergut, 2013).

Scientists K.L. Corum and J.F. Corum have modeled RF coils as slow-wave anisotropic waveguides. A solution of the boundary value problem has given not only the fields, but also the eigenvalue equation for the propagation parameter $(\tau)$, the velocity factor $(\mathrm{Vf})$, the wave effective characteristic impedance $(\mathrm{Zc})$, and the limiting voltage magnification $(1 / \alpha \mathrm{h})$ caused by wave interference or cavity modes. These parameters permit a comprehensive engineering description of helix design as a simple surface-wave transmission line, and they are appropriate for designing and tuning Tesla coil helical resonators on conventional Smith charts. Further, this development analytically clarifies the smooth conceptual transition from field theory - to distributed elements to lumped elements (Corum, K.L. \& Corum, J.F., 2001).

Scientists Hudyakov G.I. has developed ideas and formulas of analytical geometry for spherical surface of the Earth globe in relation to main tasks of global geodesy and navigation. It examines peculiarities of sphere inner geometry and properties of its primary, secondary and higher-order curves. It was proved that spherical hyperbola and parabola are spherical ellipses with specific parameters. The Cartesian ordinates were introduced into the sphere and the relation between them and polar spherical coordinates was established. With the help of central projection of sphere points on tangential plane the corresponding elliptical plane with beltrami ordinates was introduced. The article describes main formulas of analytical geometry for projected elliptical plane, which correspond to geometry of projected sphere. It also introduces several formulas for primary, secondary and higher-order curves for this sphere (Hudyakov, 2017).

\section{MATERIALS AND METHODS}

Any line projection system consists of rectilinear or curvilinear rays, which collectively represent the set $\infty 2$ (congruence). For the formation of surfaces assets of $\infty 1$ rays that project some line, congruencies with the property of passing a single ray are best all the way through a point of space that is not congruent with respect to this congruence (Darboux, 1914). For algebraic rays, it is said that the congruence that they form is of the first order. As already noted, three functions of three variables (Simenko \& Voronina, 2017)

$$
x=f(t, u, v), y=\varphi(t, u, v), z=\psi(t, u, v)
$$

represent not only a congruence of lines, but also a system of curvilinear coordinates. This means that when deriving parametric equations of some congruence of lines (1), this equation corresponds to two congruencies of other lines, depending on which of the three independent variables $t, u$ or $v$ we take as the parameter the position of the point on the ray of the corresponding congruence. In other words, representing the functions (1) as the determinant of the coordinate system, we must ensure that these functions pass through the point of parametrization by the space of three coordinate surfaces and three coordinate lines as pairwise intersections of coordinate surfaces. For example, functions that define a cylindrical coordinate system.

$$
x=v, y=u \cos t, z=u \sin t
$$


also specify three congruencies of coordinate lines; $\mathrm{t}$ - lines, as cross-sections of coordinate cylinders $u=$ const with coordinate planes $v=$ const , which form a congruence of circles with centers on the axis $O X$ and planes perpendicular to $O X ; u$ - lines, the congruence of the lines of intersection of coordinate planes (plane of the beam with the $O X$ axis) with coordinate planes $t=$ const (planes perpendicular to the $O X$ axis); $v$ - lines parallel to the $O X$ axis, as sections of coordinate cylinders $u=$ const coaxial with $O X$, with coordinate planes $t=$ const belonging to the pencil with the $O X$ axis. It is for this reason that curvilinear projection is often associated with the congruence of straight lines (Ivzhenko, 1996; Kotov, 19691; Kotov, 19692). Systems of curvilinear projection, considered in the article are widely known. Here, the goal is to compile their analytical model from the standpoint of the general analytic theory of surface formation by the method of analytical modeling of the formation of surfaces by nonlinear projection (Darboux, 1914).

\section{RESULTS}

Consider the congruence of the right cylindrical helical lines with the common axis OZ, with a constant pitch $\mathrm{h}$, whose parameters are: $\mathrm{v}$ is the radius of the incidence cylinder, and $\mathrm{u}$ is the angular parameter of the position of the helical line on the incidence cylinder. The angle $\mathrm{u}$ is measured from the half-plane XOZ to the half-plane $\mathrm{AOZ}$ under the conditions of the location of the point $\mathrm{A}$ on the incidence cylinder section by the XOY plane. For the position parameter of the point on the helical line, take the angle t, the count of which will be from the half-plane $\mathrm{AOZ}$ to the half-plane MOZ, representing M as the current point. The direction of reference of both the angle $u$ and the angle $t$ is counter-clockwise.

$$
x=v \cos (u+t), y=v \sin (u+t), z=\frac{h t}{2 \pi}+z_{0}
$$

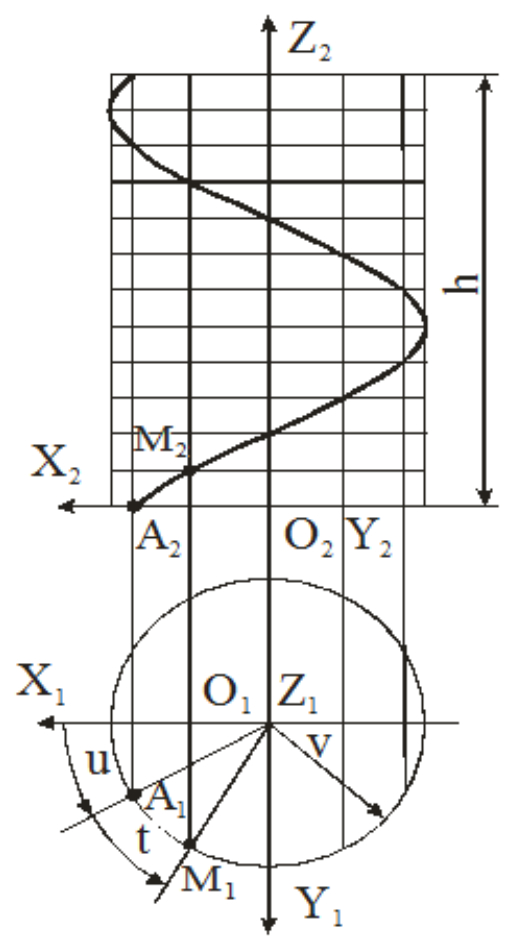

Fig. 1. The congruence parameters of cylindrical helical lines

The selected content of the congruence parameters of the helical lines corresponds to the parametric equations.

We define the ray of screw projection passing through an arbitrary point $M\left(x_{M}, y_{M}, z_{M}\right)$.. For this it is necessary, using the parametric equations (3), to express the parameters $t_{M}, u_{M}, v_{M}$ конгруэнции of the congruence in terms of the coordinates $x_{M}, y_{M}, z_{M}$ of $M$. From the first two equations we obtain

$$
v_{M}=\sqrt{x^{2}{ }_{M}+y^{2}{ }_{M}} \text {. }
$$

From the third equation we obtain

$$
t_{M}=\frac{2 \pi\left(z_{M}-z_{0}\right)}{h} .
$$

We cannot determine $u$ by $x_{M}, y_{M}, z_{M}$ by one expression. Therefore, we represent $u$ by such expressions: 


$$
\begin{aligned}
& x=0, \quad y>0 \Rightarrow \quad u_{M}=\frac{\pi}{2}-\frac{2 \pi\left(z_{M}-z_{0}\right)}{h} ; \\
& x=0, \quad y<0 \Rightarrow \quad u_{M}=\frac{3 \pi}{2}-\frac{2 \pi\left(z_{M}-z_{0}\right)}{h} ; \\
& x>0, \quad y>0 \Rightarrow \quad u_{M}=\operatorname{arctg} \frac{y_{M}}{x_{M}}-\frac{2 \pi\left(z_{M}-z_{0}\right)}{h} ; \\
& x>0, \quad y<0 \Rightarrow \quad u_{M}=\operatorname{arctg} \frac{y_{M}}{x_{M}}+2 \pi-\frac{2 \pi\left(z_{M}-z_{0}\right)}{h} ; \\
& x<0 \Rightarrow \quad u_{M}=\operatorname{arctg} \frac{y_{M}}{x_{M}}+\pi-\frac{2 \pi\left(z_{M}-z_{0}\right)}{h} ; \\
& t_{M}=\frac{2 \pi\left(z_{M}-z_{0}\right)}{h} ; \quad v_{M}=\sqrt{x_{M}{ }^{2}+y_{M}{ }^{2}} .
\end{aligned}
$$

Thus, the expressions (4) uniquely determine the ray (or rather its parameters) passing through a fixed point of space. This indicates that the congruence of the cylindrical helical lines (3) is a first order congruence. According to the general theory of analytic surface shaping (Skidan, 2001; Skidan, 2002), any congruence surface (3) can be obtained as a family of helical rays that project a certain line of space. It is recommended that this line be given by an equation that is internal with respect to equation (3).

\section{Discussions}

Consider examples of obtaining analytical and computer models of known helical surfaces that are studied in courses of descriptive and differential geometry.

Example 1. Compose the internal and parametric equations of a direct (minimal) helicoid, provided it passes through the point $M(3,4,2)$. The pitch of the helicoid is $h=10$.

Decision. Using formula (4), calculate the value of $u_{M}$ by substituting the coordinates of point $\mathrm{M}$ and $z_{0}$ $=0$.

$$
u_{M}=\operatorname{arctg} \frac{y_{M}}{x_{M}}-\frac{2 \pi\left(z_{M}-z_{0}\right)}{h}=\operatorname{arctg} \frac{4}{3}-\frac{2 \cdot 3,14 \cdot 2}{10}=-0,33
$$

Equation (5) is the internal equation for the desired direct (minimal) heliocoid passing through the point M. We obtain the parametric equations by substituting (5) in (3)

$$
x=v \cos (-0,33+t), \quad y=v \sin (-0,33+t), \quad z=\frac{5 t}{\pi} .
$$

Fig. 2 shows the compartment of the surface (6) corresponding to the values $-5 \leq v \leq 5$.

Example 2. Compose the internal and parametric equations of an oblique helix, the straight-line generator of which is inclined to the axis $O Z$ at an angle of $150^{\circ} \frac{5 \pi}{6}$, the pitch of the helicoid $h=10$.

Decision. We show the generator of an oblique helicoid for $a=0, t=0$ in the projection onto the plane ZOX (Fig. 3). From the variable $\mathrm{v}$ we go to the variable $w$. The transition function $v=w \sin \left(\frac{5 \pi}{6}\right)$, where $w$ is the length of the segment from the origin to the point $M$ on the generator.

The internal equation of the surface will be

$$
v=w \sin \left(\frac{5 \pi}{6}\right), \quad z_{0}=w \cos \left(\frac{5 \pi}{6}\right), \quad u=0 .
$$




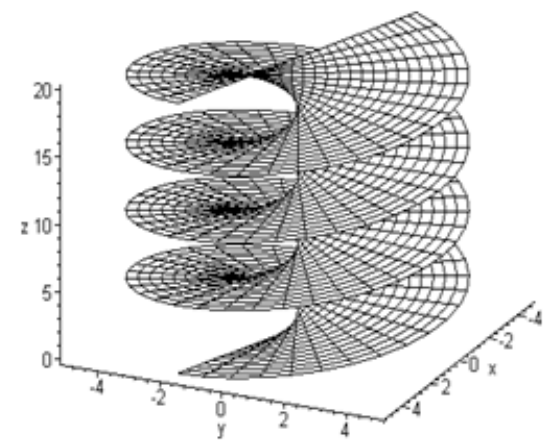

Fig. 2. The direct (minimal) helicoid

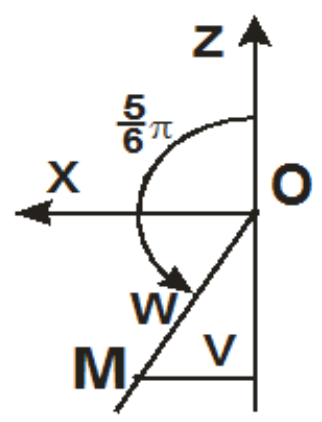

Fig. 3

Substituting in (3) for $\mathrm{v}$ its expression from (7) and adding to the expression $\mathrm{z}$ the application of $\mathrm{z}_{0}$ from (7), we obtain the parametric equations of the required surface. Moreover, $u=0$.

$$
x=w \sin \left(\frac{5 \pi}{6}\right) \cos t, \quad y=w \sin \left(\frac{5 \pi}{6}\right) \sin t, \quad z=w \cos \left(\frac{5 \pi}{6}\right)+\frac{h t}{2 \pi} .
$$

Fig. 4 shows the surface of an oblique helicoid with parameters $0 \leq w \leq 5,0 \leq t \leq 4 \pi$.

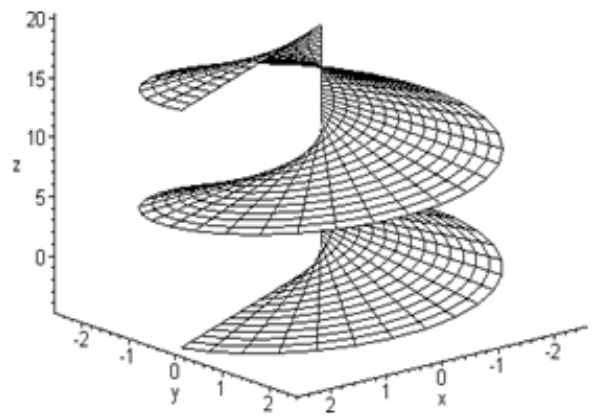

Fig. 4. Skew helicoid

As in the first example, we could put an additional condition for passing through the surface through a fixed point $M\left(x_{M}, y_{M}, z_{M}\right)$. But in this case, to determine $u_{M}$, it would be necessary to compute

than

$$
w_{M}=\frac{\sqrt{x_{M}^{2}+y_{M}{ }^{2}}}{\sin \left(\frac{5 \pi}{6}\right)}
$$

$$
t_{M}=\frac{2 \pi\left(z_{M}-w_{M} \cos \left(\frac{5 \pi}{6}\right)\right)}{h}
$$

and, finally, $u_{M}$.

Thus, in the first two equations (8), instead of the argument $t$, the argument is $u_{M}+t$. 
Example 3. Compose the internal and parametric equations of the Dini helicoid, whose step is $h=10$, and the parameter $a=5$. tractris

Decision. The internal equation of the Dini helicoid is obtained as the parametric equations of the

$$
v=a \sin w, \quad z_{0}=a\left(\ln \operatorname{tg} \frac{w}{2}+\cos w\right), \quad u=0 .
$$

We substitute their expressions (9) in place of $v$ and $z_{0}$ in (3). We obtain the parametric equations of the Dini helix in the form

$$
x=a \sin w \cos t, \quad y=a \sin w \sin t, \quad z=a\left(\ln t g \frac{w}{2}+\cos w\right)+\frac{h t}{2 \pi}
$$

In Fig. 5 shows a section of the Dini Helix corresponding to

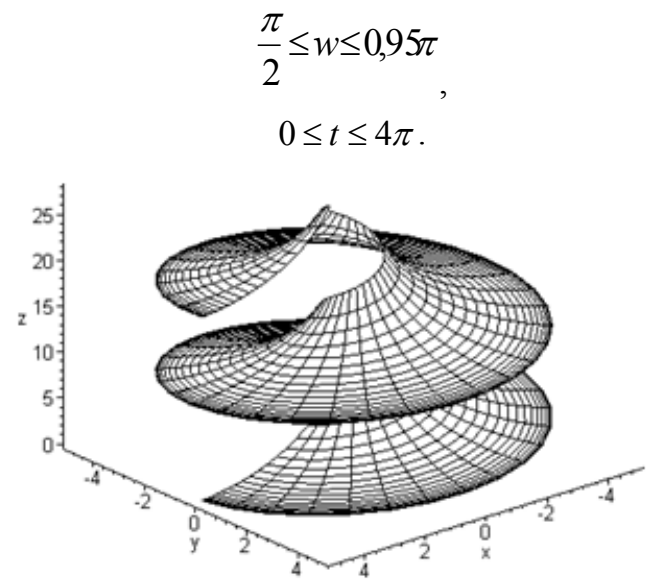

Fig. 5. Helix Dini

Example 4. Compose the internal and parametric equations of a helical surface obtained by screw projection of an ellipse.

$$
x=\frac{a b \cos u}{\sqrt{a^{2} \sin ^{2} u+b^{2} \cos ^{2} u}}, \quad y=\frac{a b \sin u}{\sqrt{a^{2} \sin ^{2} u+b^{2} \cos ^{2} u}}, \quad z=0
$$

under the condition $a=6, b=3, h=10$.

Decision. The parametrization of the ellipse (11) is such that $\mathrm{u}$ is the angle between the $O X$ axis and the radius vector of the point on the ellipse. Hence it follows that the parameter $u$ of equation (11) must be taken as the parameter $u$ of the position of the helical line passing through the point of the ellipse.

By the expression with (4), we define

$$
v=\sqrt{x^{2}+y^{2}}=\frac{a b}{\sqrt{a^{2} \sin ^{2} u+b^{2} \cos ^{2} u}}
$$

- the internal equation of the desired helical surface.

Substituting (12) into (3), taking into account $z 0=0$, gives

$$
\begin{aligned}
& x=\frac{a b}{\sqrt{a^{2} \sin ^{2} u+b^{2} \cos ^{2} u}} \cos (u+t), \\
& y=\frac{a b}{\sqrt{a^{2} \sin ^{2} u+b^{2} \cos ^{2} u}} \sin (u+t), \quad z=\frac{h t}{2 \pi} .
\end{aligned}
$$

The values correspond to the values

$$
\begin{gathered}
0 \leq u \leq 2 \pi, \\
0 \leq t \leq 2 \pi
\end{gathered}
$$




\section{CONCLUSION}

Analytical interpretation of the method of shaping by screw projection allows to take into account such requirements to the surface as passing through a point or line, and, what is most important, to use computer technologies in the design and manufacture of screw-shaped products on CNC-controlled equipment. Computer visualization of surfaces and special lines on them, on the one hand, demonstrate a unique adaptability to modern computer graphics software, and on the other hand it is an effective criterion for the reliability of the results obtained.

\section{RECOMMENDATIONS}

The materials of this article can be useful for graduate students, teachers studying constructive models of non-traditional projection systems. In the process of research, new questions and problems appeared that needed their solution. It is necessary to continue research into development of a general algorithm for the construction of parametric equations of the set (congruence) of rays of non-traditional projection systems and their projecting surfaces and apply it to systems for projecting congruencies of co-axial conical helical lines of a constant step of a constant inclination.

\section{REFERENCES}

[1] Artemyev, V.G., Gubeydullin, H.H., Kurdyumov,V. I., Voronina, M.V. (2010). Calculation, manufacture and use of springs for different purposes: Monograph. Ulyanovsk: Ulyanovsk state agricultural academy named after P.A. Stolypin, 221 p.

[2] Artemyev, V.G., Gubeydullin, H.H., Voronina, M.V., Shigapov I.I. (2012). Design, manufacture and use of springs of different purposes: Monograph. Ulyanovsk: Technological institut - Academy branch of Ulyanovsk state agricultural academy named after P.A. Stolypin, $233 \mathrm{p}$.

[3] Artemyev, V.G., Artiushin, A.A., Reznik, E.I., Igonin, V.N., Gubeydullin, H.H., Isaev, J. M., Kushnarenko, I. G., Voronina, M.V., Gaisin R.M. (2004). Springs-transporting bodies of agricultural machinery. Theory and practice. Ulyanovsk: Ulyanovsk state agricultural academy named after P.A. Stolypin, 554 p.

[4] Artemyev, V.G., Kushnarenko, I.G., Voronina, M.V., Shagunov, F.G., Pylin, A.G., Dumbolov, D.U. (1999). A device for pumping high-viscosity liquids. Patent RUS 2176033, 05.05.1999

[5] Isaev, J. M., Artemyev, V.G., Voronina, M.V., Nazarova, N.N. (2009). Planting unit. Patent RUS 91797, 20.10.2009.

[6] Baroiu, N., Berbinschi, S., Teodor, V., Oancea, N. (2012). The modeling of the active surfaces of a multi-flute helical drill with curved cutting edge using the sv\& toolbox environment. ICT-2012 13th International Conference on Tools, 27 - 28 March 2012, Miskolc, Hungary.

[7] Berbinschi, S., Teodor, V., Oancea, N. (2011). A 3D method for profiling the shaping tool for the generation of helical surfaces. The annals of "Dunărea de Jos" University of GALAŃI, Manufacturing Science and Engineering Department, ISSN 1221- 4566, GALAŃI, Romania, pp. $19 \ldots 24$.

[8] Bergman, D.L. \& Refael, G. (2010). Bulk metals with helical surface states. Physical review B82, pp.195417-1...195417-11.

[9] Corum, K.L. \& Corum, J.F.(2001). RF Coils, Helical Resonators and Voltage Magnification by Coherent Spatial Modes. TELSIKS 2001, University of Nis, Yugoslavia and MICROWAVE REVIEW, pp.1...10.

[10] Ćuković, S., Devedžić, G., Ghionea, I. (2009). Automatic determination of grinding tool profile for helical surfaces machining using catia/vb interface U.P.B. Sci. Bull., Series D, 72 (2), pp. 85...96.

[11] Darboux, G. (1914). Lecons sur la theorie generale des surfaces, Paris, vol. 4, p. 576.

[12] Ergut, M., Korpinar, T., Turhan, E. (2013). On normal ruled surfaces of general helices in the sol space, TWMS J. Pure Appl. Math., 4 (2), pp. $125 \ldots 130$

[13] Folomkin, A.I. \&Voronina, M. V., (2017). Educational library for the automation of design calculations springs using SOLIDWORKS API. Man In India, 97 (3), pp. 443...451.

[14] Frumusanu, G., Berbinschi, S., Oancea, N. (2013). Cylindrical surfaces enwrapping helical surfaces rack-tool versus planning tool. Proceedings in Manufacturing Systems, ISSN 2067-9238, 8 (3), pp. 153...158.

[15] Han, Quan-Quan \& Liu, Ri-Liang (2013). Mathematical Model and Tool Path Calculation for Helical Groove Whirling. Research Journal of Applied Sciences, Engineering and Technology, 6 (1), pp. $3584 \ldots 3587$.

[16] Hudyakov, G.I. (2017). Development of methods of analytical geometry of a sphere for solving geodesy and navigation tasks. Journal of Mining Institute. Vol. 223, pp.70...82. DOI http://dx.doi.org/10.18454/pmi.2017.1.70.

[17] Ivzhenko, A. V. (1996). Designing surfaces by separating them from congruences of parabolas. Collection of works of the III International Scientific and Practical Conference "Modern Problems of Geometric Modeling", Melitopol, Ukraine, vol. II, pp. $231 \ldots 232$.

[18] Kotov, I.I. (1969)1. Instant algebraic transformations and their possible applications. Cybernetics of graphics and applied geometry of surfaces: Proceedings of the MAI, Moscow, 3, pp. $71 \ldots 83$.

[19] Kotov, I.I. (1969)2. Instantaneous transformations and vector methods for constructing surfaces. Cybernetics of graphics and applied geometry of surfaces: Proceedings of the MAI, Moscow, 3, pp. 27...33.

[20] Krivoshapko, S.N. \& Bock Hyeng, Ch. A. (2012). Classification of cyclic surfacesand geometrical research of canal surfaces. IJRRAS, $12(3)$, pp. $360 \ldots 374$.

[21] Krivoshapko, S.N. \& Ivanov, V. N. (2015) Helical Surfaces. In: Encyclopedia of Analytical Surfaces. Springer, Cham, pp. 225 ...258.

[22] Krivoshapko, S.N. \& Rynkovskaya, M. (2017). Five Types of Ruled Helical Surf aces for Helical Conveyers, Support Anchors and Screws. MATEC ICMME 2016, Web of Conferences, pp. 1...5.

[23] Olejníková, T. (2009). Helical Two-Revolutional Cyclical Surface. Acta Polytechnica Hungarica, 6, 4, pp.101...110.

[24] Olejníková, T. (2014). Cyclical Surfaces Created by Helix on Torus. American Journal of Applied Mathematics, 2 (6), 2014, pp. 204...208.

[25] Podgorny, A. L., 1963, "Screw design". Proceedings of general theoretical chairs of the Ukrainian Agricultural Academy. Kiev, Ukraine.

[26] Popa, C. et al. (2014). Side Mill Tool Profiling for Generation of Helical Surfaces Determined by Reverse Engineering. Applied Mechanics and Materials, 657, pp. 28...32.

[27] Pottmann, H., Leopoldseder, S., Wallner, J., Peternell, M. (2002). Recognition and reconstruction of special surfaces from point clouds. Proceedings of the ISPRS Commission III, Vol.34, Part 3A « Photogrammetric Computer Vision», Graz, pp. 271 ...276. 
[28] Podgorny, A. L. (1963). Screw design. Proceedings of general theoretical chairs of the Ukrainian Agricultural Academy. Kiev, Ukraine, pp. 228...234.

[29] Simenko E. V. \& Voronina M. V. (2017). Constructive methods of forming surfaces. International Journal of Applied Engineering Research, 12 (6), pp. $956 \ldots 962$.

[30] Skidan, I. A. (2001). The Analytic Theory of the Applied Formuational Principle on the Basis of the Global Parametrization. Applied Geometry is the engineering graph. Pratsi / Tavriys'kai sivivna agrotechnic academia. Melitopol: TDATA, 13, 4, pp. $22 \ldots 28$.

[31] Skidan I.A. (2002). Generalization of Analytical Formation Methods Founded on Global Parametrization of Surfaces. The Applied Geometry and Engineering Graphics. KNUBA.: Kiev, Ukraine, 70, pp. $79 \ldots 84$.

[32] Teodor, V., Ionuţ, P., Gabriel, D., Nicolae, O. (2009). The profiling of disk tool for generation of discreetly known helical surfaces. The annals of "dunărea de jos" University Of Galaţi Fascicle V, Technologies in machine building, ISSN 1221- 4566, pp. 53 ...58.

[33] Tevlin, A. M. (1968). Screw design and its application for solving geometric and technical problems. News of higher educational institutions of the USSR. Mechanical engineering, No.2.

[34] Tevlin, A. M., Ivanov J.N., Podkritov A.N. (1966). Profiling of conjugate screw surfaces by the method of curvilinear design. Questions of applied geometry. Collection of works of graduate students and applicants. Moscow: MAI, pp. $6 \ldots 16$.

[35] Znamenskiy, D., Tuan, Khan Le, Poupon, A., Chomilierand, J., Mornon, J.-P. (2000). $\beta$ Sheet modeling by helical surfaces. Protein Engineering. 13 (6), pp. $407 \ldots 412$.

\section{AUTHOR PROFILE}

Elena Vasilyevna Simenko PhD in Engineering, Candidate of Technical Sciences, Associate Professor, Department of Descriptive Geometry and Graphics, Saint-Petersburg Mining University, 2, 21st Line, Saint Petersburg, 199106, Russian Federation, e-mail: kii-simenko@mail.ru

Sergei Anatolyevich Ignatiev Head of Descriptive Geometry and Graphics Department, PhD in Engineering, Candidate of Technical Sciences, Associate Professor, Saint-Petersburg Mining University, 2, 21st Line, Saint Petersburg, 199106, Russian Federation, e-mail: isa@spmi.ru

Marianna Vladimirovna Voronina* $\mathrm{PhD}$ in Engineering, Candidate of Technical Sciences, Associate Professor, Department of Descriptive Geometry and Graphics, Saint-Petersburg Mining University, 2, 21st Line, Saint Petersburg, 199106, Russian Federation, e-mail: maria.vv@mail.ru

\footnotetext{
* Correspondent Author
} 